\title{
Effect of Intravenous Lidocaine on Postoperative Pain in Patients Undergoing Intraspinal Tumor Resection: Study Protocol for a Prospective Randomized Controlled Trial
}

This article was published in the following Dove Press journal:

Journal of Pain Research

\section{Hongli Yue* \\ Man Zhou (D* \\ Yu Lu \\ Liang Chen \\ Weihua Cui (D)}

Department of Anesthesiology, Beijing Tian Tan Hospital, Capital Medical University, Beijing, People's Republic of China

*These authors contributed equally to this work
Correspondence: Weihua Cui Department of Anesthesiology, Beijing Tian Tan Hospital, Capital Medical University, No. I19, Nan Si Huan Xi Lu, Fengtai District, Beijing 100070, People's Republic of China

Tel +8613651315600

Fax +86I059976658

Email weihuacui@ccmu.edu.cn
Purpose: Patients undergoing intraspinal tumor resection usually experience severe acute pain, delaying postoperative rehabilitation, and increasing incidence of chronic pain. Recently, an increasing number of studies have found that low-dose intravenous lidocaine infusion during and/or after surgery can reduce opioid usage and the incidence of related side effects, inhibit hyperalgesia and promote recovery. Thus far, no studies have evaluated the analgesic effect and safety of perioperative intravenous lidocaine infusion for intraspinal tumor resection, especially the long-term analgesic effects of patient-controlled analgesia (PCA) with lidocaine during the first postoperative 48 hours. This study tests the hypothesis that intra- and postoperative systemic lidocaine infusion for patients undergoing intraspinal tumor resection can relieve postoperative acute or chronic pain and reduce the opioid dosage and incidence of related side effects without other problems.

Study Design and Methods: This is a prospective, randomized, placebo-controlled, and double-blinded study. In total, 180 participants scheduled for intraspinal tumor resection will be randomly divided into lidocaine and placebo groups. The lidocaine group will be administered lidocaine intravenously during anesthesia and postoperative pain management during the first 48 postoperative hours; the placebo group will be administered normal saline at the same volume, infusion rate, and timing. The primary outcome will be the postoperative visual analog scale (VAS) score. Secondary outcomes will be postoperative cumulative sufentanil consumption, indicators of postoperative recovery, and the incidence of perioperative adverse events.

Discussion: This study investigates the effect of continuous intravenous lidocaine infusion on postoperative sufentanil consumption and VAS scores. The findings will provide a new strategy of anesthesia and analgesia management for intraspinal tumor resection.

Keywords: postoperative pain, intraspinal tumor resection, lidocaine, opiates

\section{Background}

Patients undergoing intraspinal tumor resection usually experience extreme pain, and without adequate postoperative analgesia, even their movement in bed can be limited due to pain. Severe tissue damage and stimulation to the sensory nerve contribute mainly to pain; another reason for severe pain is likely the large dose of intraoperative opioids administered. Previous studies have shown that opioids induce hyperalgesia, which enhances the perception of pain. ${ }^{1}$ As electrophysiological monitoring is necessary for 
this type of operation, muscle relaxants are forbidden in the maintenance of anesthesia, and only large doses of opioids can be used to avoid intraoperative body movement. Because of the above reasons, patients undergoing intraspinal tumor resection experience severe postoperative pain that requires a large amount of analgesics, including opioids, which is not beneficial for rehabilitation. ${ }^{2,3}$ We hope to find a new anesthesia and analgesia strategy to reduce perioperative opioid dosage, alleviate postoperative pain, promote patient recovery, shorten hospital stays and reduce medical costs.

Lidocaine is a versatile anesthetic that can be used for local anesthesia, nerve blocks, intrathecal anesthesia, general anesthesia and the long-term treatment of ventricular arrhythmias. In recent years, increasing numbers of studies have found that low-dose lidocaine intravenous infusion during and/or after surgery can reduce the dosage of opioids, inhibit hyperalgesia, alleviate postoperative acute or chronic pain, reduce nausea and vomiting, shorten the duration of intestinal paralysis, inhibit the inflammatory reaction, shorten the length of hospital stay and reduce postoperative cognitive dysfunction. ${ }^{4-6}$ Thus far, studies of general anesthesia with intravenous lidocaine infusion have mainly concentrated on digestive, ${ }^{3,7-13}$ urogenital, ${ }^{14,15}$ breast, $^{16}$ thoracic and cardiovascular ${ }^{17,18}$ and ambulatory surgeries. ${ }^{19}$ Conversely, there is no report on intravenous lidocaine for intraspinal tumor resection. Can lidocaine still show satisfactory analgesic effects in these patients with a high intensity of postoperative pain? Is lidocaine equally beneficial to the recovery of gastrointestinal function in such nonabdominal surgery? Does lidocaine have no effect on the monitoring of somatosensory evoked potential and motor evoked potential in this type of operation? All of these questions need to be answered by rigorously designed clinical trials.

Based on the results of existing studies regarding the use of intravenous lidocaine infusion in a variety of surgeries, we hypothesized that intravenous lidocaine infusion during and after intraspinal tumor resection can alleviate acute and chronic pain caused by tissue damage, nerve stimulation and opioid-induced hyperalgesia, reduce the incidence of opioid-related side effects, and hasten the recovery of patients.

Accordingly, a single-center, prospective, randomized, placebo-controlled, double-blinded study was designed to evaluate the analgesic effect and safety of perioperative intravenous lidocaine infusion for intraspinal tumor resection. The primary outcome will be the postoperative pain score. Secondary outcomes will include postoperative cumulative sufentanil consumption, indicators of postoperative recovery, and the incidence of perioperative adverse events.

\section{Study Design and Methods}

This study is a single-center, prospective, randomized, placebo-controlled and double-blinded clinical trial designed to compare the efficacy and safety of intravenous lidocaine infusion during and after intraspinal tumor resection. The patients will be enrolled consecutively in Beijing Tian Tan Hospital, Capital Medical University, China. The patient flow chart for the study is shown in Figure 1, and the trial schedule is presented in Table 1. Patients will be screened, recruited and followed-up by three investigators. All researchers will be trained using the same protocol.

\section{Approval of the Study Protocol}

All procedures in the trial will be conducted in accordance with the World Medical Association's “Helsinki Declaration (version 19 October 2013)". The study plan (protocol version 1.0) was approved by the Ethics Committee of Beijing Tian Tan Hospital, Capital Medical University, China (KY2017063-02). This study protocol has been registered at chictr.org. cn (ChiCTR1900021134).

\section{Informed Consent}

All participants in this trial will sign informed consent documents. Moreover, all participants will be given sufficient time to decide whether to participate in this study. Patients who participate in the study will have the right to obtain all relevant information, and they will be allowed to withdraw their consent or discontinue participation without restrictions at any time during the study. The confidentiality of participant records will be protected.

\section{Study Population}

\section{Inclusion Criteria Will Be as Follows}

- Patients scheduled for elective intraspinal tumor resection in the thoracic or lumbosacral segments under general anesthesia;

- American Society of Anesthesiologists (ASA) physical status I-II;

- Age 18-65 years.

\section{Exclusion Criteria Will Be as Follows}

- History of drug abuse, psychiatric disorders, or obesity ( $>130 \%$ standard weight);

- History of cardiovascular diseases and unstable hypertension; 


\section{Enrollment}

Assess for eligibility

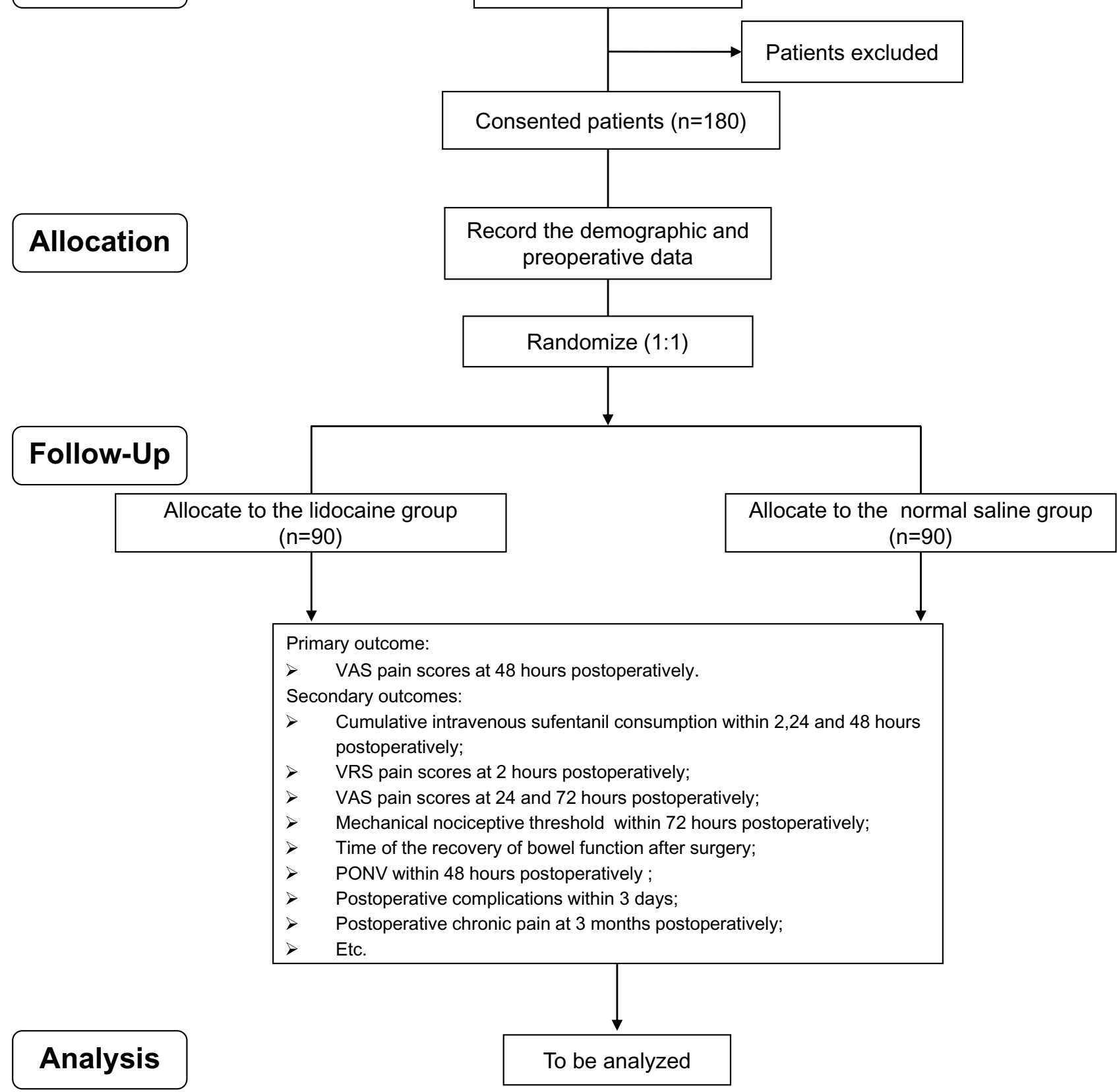

Figure I Flowchart of the study procedure.

Abbreviations: VAS, visual analog scale; VRS, verbal rating scale; PCA, patient-controlled analgesia; PONV, postoperative nausea and vomiting.

- History of chronic upper limb pain or sensory disorders;

- Communication disorders;

- Contraindication for propofol, opioids and lidocaine (eg, allergy, heart block, porphyrin disease);

- Difficulty understanding the meaning of the visual analog scale (VAS, $0=$ no pain, $10=$ most severe pain you can imagine) before surgery;
- Failure to use a patient-controlled analgesia (PCA) device.

\section{Randomization and Blinding}

The participants will be randomized to the lidocaine and placebo (control) groups at a 1:1 ratio. Randomization will be performed with a random number list generated by a computer. Opaque sealed envelopes containing the participant's order on 
Table I Trial Schedule

\begin{tabular}{|c|c|c|c|c|c|c|c|c|}
\hline \multirow[t]{3}{*}{ Time point } & \multicolumn{8}{|l|}{ Study Period } \\
\hline & \multirow{2}{*}{$\begin{array}{l}\text { Enrollment } \\
\text { Preoperative }\end{array}$} & \multirow{2}{*}{$\begin{array}{l}\text { Allocation } \\
\text { Od }\end{array}$} & \multicolumn{6}{|c|}{ Post-Allocation } \\
\hline & & & Surgery & $2 \mathbf{h}$ & Id & $2 d$ & 3d & $3 m$ \\
\hline \multicolumn{9}{|l|}{ Enrollment } \\
\hline Eligibility screening & $x$ & & & & & & & \\
\hline Informed consent & $x$ & & & & & & & \\
\hline Allocation & & $x$ & & & & & & \\
\hline \multicolumn{9}{|l|}{ Interventions } \\
\hline Lidocaine & & $x$ & & & & & & \\
\hline Normal saline (NS) & & $x$ & & & & & & \\
\hline \multicolumn{9}{|l|}{ Assessments } \\
\hline Baseline variables & $x$ & $x$ & $x$ & & & & & \\
\hline Intraoperative data & & & $x$ & & & & & \\
\hline Cumulative sufentanil consumption & & & & $x$ & $x$ & $x$ & & \\
\hline PCA press counts & & & & & $x$ & $x$ & & \\
\hline VRS score & & & & $x$ & & & & \\
\hline VAS score & $x$ & & & & $x$ & $x$ & $x$ & $x$ \\
\hline Mechanical threshold & $x$ & & & & $x$ & $x$ & $x$ & \\
\hline PONV & & & & & $x$ & $x$ & $x$ & \\
\hline Data on return of bowel function & & & & & $x$ & $x$ & $x$ & \\
\hline Total rescue medication usage & & & & & $x$ & $x$ & $x$ & $x$ \\
\hline Postoperative complications & & & & & $x$ & $x$ & $x$ & $x$ \\
\hline
\end{tabular}

Abbreviations: VAS, visual analog scale; VRS, verbal rating scale; PCA, patient-controlled analgesia; PONV, postoperative nausea and vomiting.

the outside and the participant's group on the inside will be used to ensure that the group allocation is not disclosed. The participants, the surgeons in charge of the operation, the anesthesiologist, and the physicians performing the follow-up will be blinded to the group assignment.

Before each surgery, the opaque sealed envelope with the patient's distribution information will be opened by the research investigator, and a $50-\mathrm{mL}$ syringe labeled "research solution" consisting of $1000 \mathrm{mg}$ of lidocaine or normal saline and a patient controlled analgesia (PCA) device will be prepared by a research investigator according to the group allocation.

\section{Subject Eligibility and Identification}

All enrolled patients will be privy to all information about the objective, procedure, benefits and potential risks of the trial and will be given sufficient time to decide whether to participate in this study. Every participant will sign an informed consent document. The researcher will then complete a qualification checklist based on the items listed in the case report form (CRF) and the patient's demographic characteristics, including age (years), sex (male or female), height, weight, and ASA grade. If the patient does not meet all the inclusion criteria, he or she will be excluded. Every participant will have the right to discontinue participation at any time.

\section{Intervention}

All participants will be randomly divided into lidocaine and placebo-controlled groups. In the lidocaine group, a bolus injection of lidocaine $1 \mathrm{mg} / \mathrm{kg}$ during anesthesia induction and continuous infusion of lidocaine $2 \mathrm{mg} / \mathrm{kg} / \mathrm{h}$ during anesthesia maintenance will be administered intravenously. Postoperative pain management during the first 48 postoperative hours will involve the use of a PCA device, which will contain sufentanil $2 \mu \mathrm{g} / \mathrm{kg}$, ondansetron $16 \mathrm{mg}$ and lidocaine $15 \mathrm{mg} / \mathrm{kg}$ diluted to $150 \mathrm{~mL}$ in normal saline. In the placebo group, the same volume of normal saline will be administered during anesthesia, and the postoperative PCA device will contain sufentanil $2 \mu \mathrm{g} /$ $\mathrm{kg}$ and ondansetron $16 \mathrm{mg}$ diluted to $150 \mathrm{~mL}$ in normal saline. The solutions and the PCA devices used in the trial 
will be administered by the anesthesiologist, who will be blinded to the group allocation.

\section{Perioperative Anesthesia Management Preoperative Visit and Evaluation}

The day before the operation, the participant will be interviewed, and the degree of pain, pain VAS score, ${ }^{20}$ and use of analgesic drugs (type and dosage) will be evaluated. Mechanical pain thresholds between the first and second metacarpal bones on the dorsal side of both hands will be measured (taking the average value of the three measurements) using the Von Frey electronic pain meter (IITC Life Science Inc., Victory Blvd Woodland Hills, CA, USA). The participant will receive an explanation of how to use the PCA device.

\section{Intraoperative Anesthesia}

All patients will be anesthetized according to a standard technique. After shifting to the operating room (OT), upper limb intravenous (IV) access will be secured, and blood pressure, electrocardiogram findings, pulse oxygen saturation, the bispectral index (BIS) and electrophysiology (somatosensory evoked potential and motor evoked potential of all extremities) will be monitored. Radial artery catheterization will be performed to monitor continuous arterial pressure before anesthesia induction. Anesthesia will be induced with midazolam (Nhwa Pharma. Corporation, Xuzhou, China) $2 \mathrm{mg}$, the trial solutions (lidocaine [Double Crane Pharma. Corporation, Yuncheng, China] $1 \mathrm{mg} / \mathrm{kg}$ or an equal volume of normal saline [Double Crane Pharma. Corporation, Wuhan, China]), sufentanil (Humanwell Pharma. Corporation, Yichang, China) $0.3 \mu \mathrm{g} / \mathrm{kg}$, propofol (Fresenius Kabi, Uppsala, Sweden)1.5-3 mg/kg, and rocuronium (Xianju Pharma. Corporation, Taizhou, China) $0.3 \mathrm{mg} / \mathrm{kg}$ IV and maintained with propofol, remifentanil (Humanwell Pharma. Corporation, Yichang, China), and the trial solution (lidocaine $2 \mathrm{mg} / \mathrm{kg} / \mathrm{h}$ or an equal volume of normal saline). Sufentanil and muscle relaxants will not be given during the surgery, and the infusion rate of the trial solutions will remain unchanged. The anesthesiologist will only adjust the infusion rate of propofol and remifentanil according to the overall consideration of the needs of the operation and intraoperative monitoring data. End-tidal carbon dioxide partial pressure $\left(\mathrm{E}_{\mathrm{T}} \mathrm{CO}_{2}\right)$ will be monitored during the operation and maintained between 35 and $45 \mathrm{cmH}_{2}$ O. Ondansetron $8 \mathrm{mg}$ and tramadol $100 \mathrm{mg}$ will be administered when closure of the incision is initiated. Administration of propofol will be stopped based on assessment of the clinical situation by the anesthesiologist. Remifentanil and the trial solutions will be discontinued when the operation is finished. The endotracheal tube will be removed when tidal volume is $>6 \mathrm{~mL} / \mathrm{kg}, \mathrm{E}_{\mathrm{T}} \mathrm{CO}_{2}$ is $<45 \mathrm{cmH}_{2} \mathrm{O}$, and swallowing and cough reflexes are normal. Antihypertensive drugs and vasoactive drugs and crystalloid and colloid infusions will be given as needed by the anesthesiologists in charge of the operation to maintain stable circulation.

\section{Postoperative Pain Management}

After tracheal extubation, the participants will be shifted to the postanesthetic care unit (PACU) and closely monitored for 2 hours. The participants will be administered boluses of sufentanil $2.5 \mu \mathrm{g}$ until their VRS-4 is $\leq 1 .{ }^{20}$ If VRS-4 is $\leq 1$ or the respiratory rate is $<12 \mathrm{bpm}$, no additional sufentanil will be administered. Intravenous postoperative pain management during the first 48 postoperative hours will be achieved with a PCA device that will be prepared by a research investigator. The PCA device will be set to a background infusion of $2 \mathrm{~mL} /$ hour, a demand dose of $0.5 \mathrm{~mL}$, a lockout interval of 15 minutes, and a maximum dosage of $5 \mathrm{~mL} /$ hour. If a patient feels pain, he or she can push the PCA button repeatedly until feeling relief. If a participant's VRS-4 score is $>1$ after the maximum dosage of $5 \mathrm{~mL} /$ hour, he or she will be administered flurbiprofen axetil intravenously twice a day at most. The doses and frequency of rescue analgesics will be documented.

\section{Follow-Up}

All participants will be visited from the day before the operation to 3 postoperative months. The follow-up will include the number of times that the patient pressed the PCA button, the cumulative consumption of sufentanil, VAS scores, the mechanical pain threshold, the PONV, ${ }^{21}$ indicators of gastrointestinal function recovery, the use of additional rescue medication (type and dosage) and adverse events. The follow-up will be completed by trained interviewers who are blinded to the group allocation. A patient will be excluded from the study if any of the following situations occur: the patient has an allergic reaction, the drug infusion time is longer than 6 hours, severe cardiovascular events that cannot be addressed with symptomatic treatment occur, the test cannot be completed due to the destruction of instruments and equipment or the patient is unwilling to continue the study. 


\section{Outcomes}

The aim of the study is to evaluate the short-term and long-term analgesic effects and safety of perioperative intravenous lidocaine for patients undergoing intraspinal tumor resection.

\section{Baseline Data}

Patient characteristics: The pre-enrollment data collected will be as follows: age (years), sex (male or female), height, weight, location of tumor (intraspinal extramedullary tumors or intramedullary tumors), pre-existing pain, and preoperative mechanical threshold.

The intraoperative data collected will be as follows: duration of surgery and anesthesia, cumulative propofol dose, cumulative remifentanil dose, the time from the cessation of propofol infusion to tracheal extubation, the time from the end of surgery to tracheal extubation, the Aldrete score at tracheal extubation, and the time from tracheal extubation to an Aldrete score of 10 .

\section{Primary Outcome}

The primary outcome will be the VAS score at 48 hours after the operation.

\section{Secondary Outcomes}

The secondary outcomes will be as follows:

1. VAS pain scores within 3 days postoperatively;

2. VRS pain scores at 2 hours postoperatively;

3. Cumulative consumption of sufentanil $(\mu \mathrm{g})$ at 2,24 and 48 hours postoperatively;

4. Mechanical pain thresholds between the first and second metacarpal bones on the dorsal side of both hands according to the Von Frey electronic pain meter at 1 day before the operation and 1,2, and 3 days postoperatively;

5. Total consumption of analgesic drugs (type and dosage) except sufentanil within 1 day before the operation and at 1, 2, and 3 days postoperatively;

6. Total number of times that patients pressed the PCA button, including effective presses and ineffective presses, at 1 and 2 days postoperatively;

7. Indicators of postoperative recovery including postoperative nausea and vomiting (PONV: 0, absent; 1, nausea not requiring treatment; 2 , nausea requiring treatment; 3 , vomiting), ${ }^{25}$ the recovery of gastrointestinal functions and other complications within 72 hours postoperatively;

8. Assessment of chronic pain 3 months after the operation.

\section{Sample Size Calculation}

Based on our previous studies, the VAS scores of patients in the lidocaine group are expected to be lower than those in the control group. The sample size was calculated with the model of Test for Two Means [Differences] in PASS V.11 software (NCSS, Kaysville, Utah, USA) on the basis of the anticipated VAS score at 2 postoperative days (1.0 \pm 2.0 in the lidocaine group, $1.8 \pm 2.2$ in the control group) as the primary endpoint and the difference test as the study design. Given an alpha level of 0.05 , a beta level of 0.2 , and an additional dropout rate of $20 \%$, the total sample size required is 90 in each group. Therefore, 180 patients (90 in each group) will be enrolled in this study.

\section{Statistical Analysis}

All data will be analyzed using Statistical Product and Service Solutions (SPSS) version 22.0 (International Business Machines Inc., USA). Continuous variables, such as age, weight, height, duration of anesthesia, duration of operation, BIS, HR, BP, electrophysiology data, dose of sufentanil, VAS score, and mechanical pain threshold, will be analyzed by the Kolmogorov-Smirnov test to determine the normality of the data distribution. Normally distributed data will be analyzed with independent t-tests or one-way ANOVA between groups or repeated measures ANOVA within groups and expressed as means \pm standard deviations. Nonnormally distributed data will be analyzed using the Wilcoxon rank test and expressed as medians and interquartile ranges. Categorical data, such as sex, ASA score, type of surgery, postoperative complications, and VRS-4, will be analyzed using nonparametric tests and expressed as the numbers of patients (percentages). A P-value $\leq 0.05$ will be considered statistically significant.

\section{Reporting of Adverse Events}

All study-related adverse events will be closely monitored, and details such as the nature, severity, and treatment will be recorded on the CRF until they are resolved and the patient is stable. Whenever an adverse event occurs, it will be reported to the principal investigator immediately, and the severity, cause and consequences will be determined. All adverse events will be compared between the groups using the chi-square test or Fisher's exact test. 


\section{Discussion}

This study is a single-center, prospective, randomized, placebo-controlled and double-blinded clinical trial designed to explore the efficacy and safety of perioperative intravenous infusion of lidocaine for pain management in patients undergoing intraspinal tumor resection. The patients will be intravenously administered a bolus of lidocaine $1 \mathrm{mg} / \mathrm{kg}$ during anesthesia induction, a continuous infusion of lidocaine $2 \mathrm{mg} / \mathrm{kg} / \mathrm{h}$ during anesthesia maintenance, and PCA with lidocaine delivered via a device that will administer sufentanil $2 \mu \mathrm{g} / \mathrm{kg}$, ondansetron $16 \mathrm{mg}$ and lidocaine $15 \mathrm{mg} /$ $\mathrm{kg}$ diluted to $150 \mathrm{~mL}$ in normal saline during the first 48 postoperative hours.

Perioperative pain management is the greatest challenge in patients undergoing intraspinal tumor resection. It has been reported that severe pain in these patients primarily originates from the following three aspects. First, neuropathic pain is caused by the lesion itself and nerve stimulation during surgery. Second, hyperalgesia induced by large doses of opioids such as remifentanil must be used to satisfy intraoperative neurophysiological monitoring (IONM), but they aggravate postoperative pain. Finally, pain is caused by the severe trauma of the operation itself and the accompanying muscle and bone tissue damage.

Lidocaine is a sodium-channel antagonist that can treat neuropathic pain by inhibiting sodium channels that generate spontaneous ectopic impulses in primary afferents. ${ }^{22}$ In addition, lidocaine has anti-inflammatory effects and can attenuate the proinflammatory effects caused by surgical injury. It achieves these anti-inflammatory effects by regulating the n-methyl-d-aspartate receptor ${ }^{23}$ and reducing the plasma concentrations of the proinflammatory cytokines IL-1, Ra, IL-6, and IL-8. ${ }^{24-26}$ Moreover, clinical and experimental researchers have clarified that intravenous lidocaine infusion can alleviate neuropathic pain and hyperalgesia induced by large doses of remifentanil. ${ }^{17,22,27}$

Many clinical studies have shown that continuous intravenous lidocaine infusion during an operation can effectively alleviate acute and chronic postoperative pain. This effect is particularly obvious in patients undergoing abdominal surgery. ${ }^{3,13}$ Different levels of analgesia produced by intravenous lidocaine infusion are mentioned in 4 abdominal surgery guidelines proposed by the international ERAS Society, including elective rectal/pelvic surgery, ${ }^{28}$ gastric (partial) resection, ${ }^{29}$ pancreaticoduodenectomy ${ }^{30}$ and gastrointestinal surgery. ${ }^{31}$ Clinical studies have also shown that continuous intravenous lidocaine infusion during laparoscopic surgery can reduce postoperative VAS scores at rest and in motion. ${ }^{7,10,12}$ In a systematic review, Zhao et al confirmed that compared to pain scores in the control group, those in a group of patients receiving a $1.5 \mathrm{mg} / \mathrm{kg}$ lidocaine intravenous injection followed by a continuous infusion of lidocaine $2-3 \mathrm{mg} / \mathrm{kg} / \mathrm{h}$ decreased by 0.743 (95\% CI: 0.240 , 1.246) during the first 12 postoperative hours, 0.712 (95\% CI: $0.184,1.239$ ) during the first 24 postoperative hours, and 0.600 (95\% CI: $0.229,0.972)$ during the first 48 postoperative hours. ${ }^{32}$ Similar results were also reported for patients undergoing laparotomy and even for those undergoing colorectal surgery, and the analgesic effect of perioperative lidocaine infusion was comparable to that of epidural analgesia. ${ }^{11}$ In addition to abdominal surgery, studies on thyroidectomy, ${ }^{33,34}$ spinal surgery, ${ }^{35}$ and thoracoscopic surgery ${ }^{17}$ have confirmed that intraoperative intravenous lidocaine infusion can significantly reduce postoperative pain scores. Regardless, the significant analgesic effect of lidocaine does not occur in all types of surgery. Indeed, evidence shows that intravenous lidocaine infusion does not reduce pain due to total hip replacement, ${ }^{36}$ cardiac surgery, ${ }^{18}$ and gynecological surgery. ${ }^{14}$ Thus far, the reasons for the different analgesic effects of continuous intravenous lidocaine infusion in various types of surgery have not been identified, but studies suggest that these differences may be related to the following two factors. The first is the pain mechanism. Studies have shown that lidocaine has an obvious analgesic effect on visceral pain and inflammatory pain caused by pulling but has reduced infiltration into bone and muscle tissues. ${ }^{36}$ Thus, the analgesic effect on pain in the bone and muscles is limited, and lidocaine has a significant effect in abdominal surgery but not in orthopedic surgery. The second factor involves the degree of surgical trauma and the extent of the inflammatory response. Lidocaine has a better analgesic effect in operations that cause major trauma and strong inflammatory reactions, such as upper abdomen and chest surgeries, whereas it has a worse analgesic effect in operations with relatively minor trauma, less severe inflammatory reactions and lower levels of postoperative pain, such as gynecological surgery. ${ }^{14}$

Hyperalgesia caused by a large dose of opioids, such as remifentanil, is one of the causes of severe postoperative pain and the use of substantial analgesics. Multiple randomized controlled studies have demonstrated that in nephrectomy, ${ }^{15}$ thoracic surgery, ${ }^{17}$ spinal surgery, ${ }^{35}$ open thyroidectomy, ${ }^{33}$ open abdominal surgery and laparoscopic surgery, ${ }^{9}$ intraoperative intravenous lidocaine infusion can 
significantly reduce the consumption of opioids in the early postoperative period, especially within the first 24 hours. However, few studies have investigated the analgesic effect of perioperative intravenous lidocaine infusion on long-term postoperative pain. In a study on abdominal surgery, perioperative intravenous lidocaine infusion reportedly reduced the consumption of morphine for 24 to 72 hours after the surgery; for example, the consumption of morphine was reduced by $3.3 \mathrm{mg}$ (95\% CI: 1.7,4.8) in patients who underwent open abdominal surgery and by $7.4 \mathrm{mg}(95 \% \mathrm{CI}: 3.4,11.4)$ in those who underwent laparoscopic surgery. ${ }^{9}$

The influence of intraoperative intravenous lidocaine infusion on postoperative pain and the consumption of sufentanil after intraspinal tumor resection will be the focus of this study. To the best of our knowledge, this study will be the first to investigate the analgesic effect of continuous intraoperative intravenous lidocaine infusion on patients undergoing intraspinal tumor resection. Furthermore, this study will explore not only the short-term analgesic effects of intravenous lidocaine infusion during the operation but also the long-term analgesic effects of PCA with lidocaine during the first 48 hours after the surgery.

Safety is also one of our concerns. Few serious adverse reactions were reported in previous studies on continuous intraoperative infusion of lidocaine $1.5-3 \mathrm{mg} / \mathrm{kg} / \mathrm{h}$. Kramer et al reported that a patient with HIV who was receiving highly effective antiretroviral therapy experienced four unresponsive episodes with tachycardia, hypertension, and oxygen desaturation on the 2 nd day after the operation. ${ }^{37}$ In the proposed trial, we will strictly limit the participants to those with ASA grades I-II. At the same time, to identify and manage potential adverse events in a timely manner, all participants will be monitored closely when the study drug is administered. If the patients exhibit any adverse events associated with the experimental drugs, such as signs of systemic toxicity (headache, tinnitus, metallic taste, seizure activity and ECG irregularities), refractory hypotension and bradycardia, the drug will be stopped immediately. In addition, if the infusion time of the experimental drugs is longer than 6 hours, administration of the drugs will be stopped, and the participants will be excluded from the trial. However, we noticed that too strict inclusion and exclusive criteria may affect generalization of the conclusion. In the future, we will explore the population with higher ASA grades.

In conclusion, this single-center, prospective, randomized, placebo-controlled, double-blinded study is designed to evaluate the analgesic effect and safety of perioperative intravenous lidocaine infusion for postoperative pain in patients undergoing intraspinal tumor resection. If the results show that continuous intravenous lidocaine infusion during and after the operation can significantly decrease postoperative sufentanil consumption and VAS scores, this study may provide a new strategy for the management of anesthesia and analgesia for intraspinal tumor resections.

\section{Trial Status}

The trial was registered at chictr.org.cn on 29 January 2019 (identifier ChiCTR1900021134). The study was approved by the Institutional Review Board at Beijing Tian Tan Hospital, Capital Medical University on 31 October 2017 (reference number KY2017-063-02). The first participant was recruited on 14 May 2019, and the anticipated completion date will be July 2021.

\section{Availability of Data}

The collected data will be stored in a secure repository at Beijing Tian Tan Hospital. The datasets will be available from the chief investigator upon reasonable request.

\section{Acknowledgments}

This work was supported by grants from the following: National Natural Science Foundation of China (81870865), Beijing Natural Science Foundation (7173255), and Beijing Municipal Administration of Hospitals Incubating Program (PX2019019).

\section{Author Contributions}

All authors made substantial contributions to the conception and design, acquisition of data, or analysis and interpretation of data; participated in drafting the article or revising it critically for important intellectual content; gave final approval to the manuscript; and agreed to be accountable for all aspects of the work. Hongli Yue and Man Zhou contributed equally to this work.

\section{Disclosure}

The authors report no conflicts of interest related to this work.

\section{References}

1. Koppert W, Schmelz M. The impact of opioid-induced hyperalgesia for postoperative pain. Best Pract Res Clin Anaesthesiol. 2007;21 (1):65-83. doi:10.1016/j.bpa.2006.12.004 
2. Bajwa SJ, Haldar R. Pain management following spinal surgeries: an appraisal of the available options. J Craniovertebr Junction Spine. 2015;6(3):105-110. doi:10.4103/0974-8237.161589

3. Weibel S, Jelting Y, Pace NL, et al. Continuous intravenous perioperative lidocaine infusion for postoperative pain and recovery in adults. Cochrane Database Syst Rev. 2018;6:CD009642.

4. Li J, Wang G, Xu W, Ding M, Yu W. Efficacy of intravenous lidocaine on pain relief in patients undergoing laparoscopic cholecystectomy: a meta-analysis from randomized controlled trials. Int J Surg. 2018;50:137-145. doi:10.1016/j.ijsu.2018.01.001

5. Reeves DJ, Foster AE. Continuous intravenous lidocaine infusion for the management of pain uncontrolled by opioid medications. J Pain Palliat Care Pharmacother. 2017;31(3-4):198-203. doi:10.1080/ 15360288.2017.1313356

6. Dunn LK, Durieux ME. Perioperative use of intravenous lidocaine. Anesthesiology. 2017;126(4):729-737. doi:10.1097/ALN.0000000 000001527

7. De Oliveira GS, Duncan K, Fitzgerald P, Nader A, Gould RW, McCarthy RJ. Systemic lidocaine to improve quality of recovery after laparoscopic bariatric surgery: a randomized double-blinded placebo-controlled trial. Obes Surg. 2014;24(2):212-218. doi:10.1007/s11695-013-1077-x

8. Kranke P, Jokinen J, Pace NL, et al. Continuous intravenous perioperative lidocaine infusion for postoperative pain and recovery. Cochrane Database Syst Rev. 2015;(7):CD009642.

9. McNicol ED, Ferguson MC, Haroutounian S, Carr DB, Schumann R. Single dose intravenous paracetamol or intravenous propacetamol for postoperative pain. Cochrane Database Syst Rev. 2016;(5):CD007126.

10. Song X, Sun Y, Zhang X, Li T, Yang B. Effect of perioperative intravenous lidocaine infusion on postoperative recovery following laparoscopic Cholecystectomy-A randomized controlled trial. Int J Surg. 2017;45:8-13. doi:10.1016/j.ijsu.2017.07.042

11. Terkawi AS, Tsang S, Kazemi A, et al. A clinical comparison of intravenous and epidural local anesthetic for major abdominal surgery. Reg Anesth Pain Med. 2016;41(1):28-36. doi:10.1097/ AAP.0000000000000332

12. Tikuisis R, Miliauskas $P$, Samalavicius NE, Zurauskas A, Samalavicius R, Zabulis V. Intravenous lidocaine for post-operative pain relief after hand-assisted laparoscopic colon surgery: a randomized, placebo-controlled clinical trial. Tech Coloproctol. 2014;18(4):373-380. doi:10.1007/s10151-013-1065-0

13. Weibel S, Jokinen J, Pace NL, et al. Efficacy and safety of intravenous lidocaine for postoperative analgesia and recovery after surgery: a systematic review with trial sequential analysis. $\mathrm{Br} J$ Anaesth. 2016;116(6):770-783. doi:10.1093/bja/aew101

14. Grady MV, Mascha E, Sessler DI, Kurz A. The effect of perioperative intravenous lidocaine and ketamine on recovery after abdominal hysterectomy. Anesth Analg. 2012;115(5):1078-1084. doi:10.1213/ ANE.0b013e3182662e01

15. Wuethrich PY, Romero J, Burkhard FC, Curatolo M. No benefit from perioperative intravenous lidocaine in laparoscopic renal surgery: a randomised, placebo-controlled study. Eur $J$ Anaesthesiol. 2012;29(11):537-543. doi:10.1097/EJA.0b013e328356bad6

16. Chang YC, Liu CL, Liu TP, Yang PS, Chen MJ, Cheng SP. Effect of perioperative intravenous lidocaine infusion on acute and chronic pain after breast surgery: a meta-analysis of randomized controlled trials. Pain Pract. 2017;17(3):336-343. doi:10.1111/papr.12442

17. Cui W, Li Y, Li S, Wang R, Li J. Systemic administration of lidocaine reduces morphine requirements and postoperative pain of patients undergoing thoracic surgery after propofol-remifentanil-based anaesthesia. Eur J Anaesthesiol. 2010;27(1):41-46. doi:10.1097/ EJA.0b013e32832d5426

18. Insler SR, O'Connor M, Samonte AF, Bazaral MG. Lidocaine and the inhibition of postoperative pain in coronary artery bypass patients. J Cardiothorac Vasc Anesth. 1995;9(5):541-546. doi:10.1016/S10530770(05)80138-7
19. De Oliveira GS, Fitzgerald P, Streicher LF, Marcus RJ, McCarthy RJ. Systemic lidocaine to improve postoperative quality of recovery after ambulatory laparoscopic surgery. Anesth Analg. 2012;115 (2):262-267. doi:10.1213/ANE.0b013e318257a380

20. Ferreira-Valente MA, Pais-Ribeiro JL, Jensen MP. Validity of four pain intensity rating scales. Pain. 2011;152(10):2399-2404. doi:10.1016/j.pain.2011.07.005

21. Moreno C, Veiga D, Pereira H, Martinho C, Abelha F. Postoperative nausea and vomiting: incidence, characteristics and risk factors-a prospective cohort study. Revista Española de Anestesiología y Reanimación. 2013;60(5):249-256. doi:10.1016/j.redar.2013.02.005

22. Kim YC, Castaneda AM, Lee CS, Jin HS, Park KS, Moon JY. Efficacy and safety of lidocaine infusion treatment for neuropathic pain: a randomized, double-blind, and placebo-controlled study. Reg Anesth Pain Med. 2018;43(4):415-424. doi:10.1097/AAP.00000 00000000741

23. Hahnenkamp K, Durieux ME, Hahnenkamp A, et al. Local anaesthetics inhibit signalling of human NMDA receptors recombinantly expressed in Xenopus laevis oocytes: role of protein kinase C. $\mathrm{Br}$ J Anaesth. 2006;96(1):77-87. doi:10.1093/bja/aei271

24. Sinclair R, Eriksson AS, Gretzer C, Cassuto J, Thomsen P. Inhibitory effects of amide local anaesthetics on stimulus-induced human leukocyte metabolic activation, LTB4 release and IL-1 secretion in vitro. Acta Anaesthesiol Scand. 1993;37(2):159-165. doi:10.1111/j.13996576.1993.tb03693.x

25. Takao Y, Mikawa K, Nishina K, Maekawa N, Obara H. Lidocaine attenuates hyperoxic lung injury in rabbits. Acta Anaesthesiol Scand. 1996;40(3):318-325. doi:10.1111/j.1399-6576.1996.tb04439.x

26. Taniguchi T, Shibata K, Yamamoto K, Mizukoshi Y, Kobayashi T. Effects of lidocaine administration on hemodynamics and cytokine responses to endotoxemia in rabbits. Crit Care Med. 2000;28 (3):755-759. doi:10.1097/00003246-200003000-00025

27. Cui W, Li Y, Li S, et al. Systemic lidocaine inhibits remifentanil-induced hyperalgesia via the inhibition of cPKCgamma membrane translocation in spinal dorsal horn of rats. J Neurosurg Anesthesiol. 2009;21(4):318-325. doi:10.1097/ANA.0b013e3181 abbde 5

28. Nygren J, Thacker J, Carli F, et al. Guidelines for perioperative care in elective rectal/pelvic surgery: enhanced recovery after surgery $\left(\right.$ ERAS $^{\circledR}$ ) society recommendations. Clin Nutr. 2012;31(6):801-816. doi:10.1016/j.clnu.2012.08.012

29. Mortensen K, Nilsson M, Slim K, et al. Consensus guidelines for enhanced recovery after gastrectomy: enhanced recovery after surgery $\left(\right.$ ERAS $\left.^{\circledR}\right)$ society recommendations. Br J Surg. 2014;101 (10):1209-1229. doi:10.1002/bjs.9582

30. Lassen K, Coolsen MM, Slim K, et al. Guidelines for perioperative care for pancreaticoduodenectomy: enhanced recovery after surgery $\left(\right.$ ERAS $\left.^{\circledR}\right)$ society recommendations. Clin Nutr. 2012;31(6):817-830. doi:10.1016/j.clnu.2012.08.011

31. Feldheiser A, Aziz O, Baldini G, et al. Enhanced recovery after surgery (ERAS) for gastrointestinal surgery, part 2: consensus statement for anaesthesia practice. Acta Anaesthesiol Scand. 2016;60 (3):289-334.

32. Zhao JB, Li YL, Wang YM, et al. Intravenous lidocaine infusion for pain control after laparoscopic cholecystectomy: a meta-analysis of randomized controlled trials. Medicine (Baltimore). 2018;97(5): e9771. doi:10.1097/MD.0000000000009771

33. Choi GJ, Kang H, Ahn EJ, et al. Clinical efficacy of intravenous lidocaine for thyroidectomy: a prospective, randomized, double-blind, placebo-controlled trial. World J Surg. 2016;40 (12):2941-2947. doi:10.1007/s00268-016-3619-6

34. Choi KW, Nam KH, Lee JR, et al. The effects of intravenous lidocaine infusions on the quality of recovery and chronic pain after robotic thyroidectomy: a randomized, double-blinded, controlled study. World J Surg. 2017;41(5):1305-1312. doi:10.1007/s00268016-3842-1 
35. Kim KT, Cho DC, Sung JK, et al. Intraoperative systemic infusion of lidocaine reduces postoperative pain after lumbar surgery: a double-blinded, randomized, placebo-controlled clinical trial. Spine J. 2014;14(8):1559-1566. doi:10.1016/j.spinee.2013.09.031

36. Martin F, Cherif K, Gentili ME, et al. Lack of impact of intravenous lidocaine on analgesia, functional recovery, and nociceptive pain threshold after total hip arthroplasty. Anesthesiology. 2008;109 (1):118-123. doi:10.1097/ALN.0b013e31817b5a9b
37. Kramer ME, Holtan EE, Ives AL, Wall RT. Perioperative intravenous lidocaine infusion adverse reaction: a case report. $A \&$ \& Pract. 2019;13(3):96-98. doi:10.1213/XAA.0000000000001002

\section{Publish your work in this journal}

The Journal of Pain Research is an international, peer reviewed, open access, online journal that welcomes laboratory and clinical findings in the fields of pain research and the prevention and management of pain. Original research, reviews, symposium reports, hypothesis formation and commentaries are all considered for publication. The manuscript management system is completely online and includes a very quick and fair peer-review system, which is all easy to use. Visit http:// www.dovepress.com/testimonials.php to read real quotes from published authors. 